\title{
Ischemic preconditioning in the brain
}

\author{
Andrew Baker MD FRCPC
}

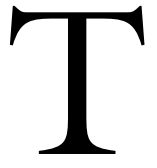

HE ability of an organism to generate physiologic responses to its environment is important for survival. Certain organs respond to sub-lethal exposures to hypoxia or ischemia by a variety of physiologic mechanisms. Some of these mechanisms induce a greater tolerance to a subsequent exposure to hypoxia or ischemia. This phenomenon is described as ischemic or hypoxic preconditioning. ${ }^{1,2}$ It is nature's "what doesn't kill you makes you stronger" message. There are a number of important biological implications of this phenomenon. First, potential therapeutic benefits of preconditioning may be recruited when physiological stresses are anticipated. Further, preconditioning is a valuable tool to study the possible mechanisms involved in cell death.

Similar to the concept that coagulation and inflammation involve a finely tuned balance between proand anti-factors, pathways leading to cell death also demonstrate this balance. The study of cell death has typically emphasized pro-factors, likely because cell death is a relatively easily-measured endpoint. Preconditioning however, is widely understood to involve the induction of cyto-protective factors and therefore is an important tool to elucidate anti-factors or innate cyto-protective mechanisms.

Examples of preconditioning are plentiful. For example, the induction of robust cyto-protective mechanisms is involved in many of the wondrous feats of nature such as prolonged submersion of diving mammals and hibernation. In clinical medicine, examples of preconditioning include the potential protective effects of pre-existing angina to the survival of patients with myocardial infarction ${ }^{3}$ or the value of short interruptions of coronary flow during percutaneous transluminal coronary angioplasty prior to the prolonged balloon inflation. ${ }^{4}$ Indeed there is now a body of literature that has established the human application of myocardial ischemic preconditioning. ${ }^{5}$
Aside from ancient remedies or possibly even homeopathy, the early demonstration of preconditioning was initially described in 1964 when it was noted that global hypoxia protected brain metabolism during subsequent hypoxia. ${ }^{6}$ Since then, the study of preconditioning has been pursued in efforts to create and understand the induction of ischemic and hypoxic tolerance. Typically, a preconditioning experiment comprises ischemia or hypoxia involving the organ of interest followed by the evaluation of the effects of subsequent exposure to ischemia or hypoxia. Interrupting certain pathways and demonstrating attenuation of tolerance would then constitute evidence that the pathway is involved in endogenous cyto-protection.

Many potential injurious pathways have been implicated in ischemic injury in the brain. Correspondingly, there are many related sources of stress other than ischemia/hypoxia that can in turn induce ischemic/hypoxic tolerance. For example, there are reports of hypothermia and hyperthermia, ${ }^{7,8}$ inflammation, ${ }^{9}$ epilepsy, ${ }^{10}$ oxygen free radicals ${ }^{11,12}$ and hyperbaric oxygen ${ }^{13,14}$ inducing ischemic or hypoxic tolerance. Because of the interwoven nature of many of the cascades involved in ischemic or hypoxic brain injury, induction of tolerance to one ischemia-related stress may be initiated through another trigger. Further, even remote preconditioning has been described, where coronary endothelial ischemic tolerance was increased following transient limb ischemia, ${ }^{15}$ presumably through an effect that modified neutrophil activation. ${ }^{16}$

In this issue of the Journal, Zhang et al. ${ }^{17}$ present a preconditioning experiment with several messages. At first take, it seems unusual to be inducing ischemic preconditioning with an enriched oxygen supply rather than a restricted one. Nevertheless, it has already been reported by this group and others that hyperoxia and hyperbaric oxygen are indeed triggers for ischemic pre-

From the Departments of Anaesthesia and Critical Care, St. Michael's Hospital and the Departments of Anaesthesia and Surgery, University of Toronto, Toronto, Ontario, Canada.

Address correspondence to: Dr. Andrew Baker, Medical Director, Trauma and Neurosurgery Intensive Care Unit, 7086 Bond Wing, St. Michael's Hospital, 30 Bond Street, Toronto, Ontario M5B 1W8, Canada. Phone: 416-864-5510; Fax: 416-864-5512; E-mail: bakera@smh.toronto.on.ca 
conditioning. Accordingly, part of the stated purpose and discussion in this report is to determine the dose of oxygen required in order to trigger preconditioning. There are several interesting extensions of their findings. That oxygen functions as a preconditioning trigger suggests that it is somehow noxious or toxic. This is consistent with the well-described problem of oxygen toxicity of the lung which is related to both concentration and duration of exposure.

The second experimental purpose and discussion topic is the investigation of the role of oxygen free radicals in the process of brain ischemic preconditioning. It not only explains the apparent paradox of hyperoxia being a trigger to preconditioning, but also highlights the importance of oxygen free radicals in brain ischemia and the innate cyto-protection possible through endogenous antioxidants.

In Zhang et al.'s report, it is suggested that preconditioning is triggered by a dose of oxygen consisting of 1 atmosphere absolute (ATA) for $24 \mathrm{hr}$ one day prior to focal ischemia. The authors introduce the notion that dosing is critical in triggering ischemic preconditioning. They point out that their previous work demonstrated that it is hyperoxia and not the hyperbaricity of oxygen that is critical. Nevertheless, these points need some clarification. The partial pressure of oxygen is the product of the environmental barometric pressure and the fraction of oxygen. The authors refer to their previous work where 2.5 ATA of $21 \%$ oxygen had no effect of triggering ischemic preconditioning, while 1 ATA of $100 \%$ did. They draw the conclusion that hyperbaricity is not important. However this was a comparison also of different doses of oxygen (partial pressures 0.5 vs 1.0 ATA). In order to sort out the environmental barometric pressure $p s$ hyperoxia question, equal partial pressures of oxygen should be compared, produced at different environmental pressures. For example: 0.60 ATA of oxygen by using 1 ATA of $60 \%$ oxygen compared with 3 ATA of $20 \%$ oxygen. Having clarified the total environmental baricity question, then dosing can be expressed by the partial pressure of oxygen (ATA achieved by any means) and the duration.

In general, dosing for this triggering effect would be of interest for various reasons. Firstly, as the authors suggest, there may eventually be therapeutic implications. Second, it is important to describe the characteristics of a research model, in order to use it effectively to study other questions. Finally, the doseresponse curve itself can tell us about the mechanism and give us perspective on the relevance of this phenomenon (preconditioning) following exposure to different doses (oxygen pressures). The report by
Zhang et al. clarifies the dose of oxygen for use with this model.

The clinical application of ischemic preconditioning is certainly attractive; however precise dosing would be critical. Using a trigger such as hypoxia, calibration of the correct dose would be difficult because of the narrow brackets between the lower threshold necessary and the upper limit of actual cell injury. It would seem that using an enriched oxygen source to trigger preconditioning would be more palatable clinically.

This study also raises the possibility that, in addition to lung toxicity, the brain may be susceptible to toxic levels of oxygen free radicals and raises questions about our use of enriched oxygen supply in other situations. For example, when weaning from cardiopulmonary bypass, a time of heightened occurrence of micro-emboli and thus potential ischemic brain injury, patients are often managed with oxygen concentrations approaching 1.0 ATA. Is this the correct time to be augmenting oxygen free radical production in the brain?

Zhang et al. present evidence to suggest that the preconditioning trigger in their study involved the production of oxygen free radicals. An oxygen free radical scavenger given to the animals during the preconditioning period prevented the phenomenon. Thus the experiment suggests that an enriched oxygen supply results in increased production of free radicals which were the trigger for the subsequent ischemic tolerance process. There are several intermediate steps. Other studies ${ }^{1}$ have demonstrated that triggers are detected by sensors which in turn employ transduction mechanisms (such as immediate early gene expression, nitric oxide, protein tyrosine phosphorylation) in order to impact effectors of preconditioning [such as heat shock proteins, free radical scavengers, gamma-amynobutyric acid (GABA) receptor upregulation and enhanced GABA release, vascular endothelial growth factor or brain derived neurotrophic factor release].

While the use of this paradigm has generated much understanding into the pathobiology of ischemic cell death, there are many questions that remain to be answered before we can proceed with clinical application in the brain. For example preconditioning mechanisms are divided into immediate and delayed phases. The myocardium demonstrates immediate preconditioning quite well. The brain in contrast responds in a delayed phase. Importantly, preconditioning-like paradigms with relatively short trigger-to-insult intervals actually result in an exacerbated brain injury. ${ }^{2}$ And to further complicate our decision to use oxygen clinically in the peri-ischemic period, animals exposed to three to six hours of 1.0 ATA of oxygen following global ischemia demonstrated a dramatically worsened mortal- 
ity. ${ }^{18}$ Thus the balance of timing, of pro- and anti-factors and of dosing is critical and complicated, just as we have learned with the manipulation of inflammation and coagulation. The inroads into the clinical application of brain ischemic preconditioning will likely follow the further elucidation of the trigger, sensor, transducer and effector mechanisms, and the potential to enhance and modulate the individual components.

\section{Préconditionnement ischémique cérébral}

La capacité d'un organisme à générer des réactions physiologiques à son environnement est importante pour la survie. Certains organes répondent à des expositions sublétales à l'hypoxie ou à l'ischémie par divers mécanismes physiologiques. Certains de ces mécanismes induisent une plus grande tolérance à une exposition subséquente à l'hypoxie ou à l'ischémie. Ce phénomène est décrit comme un préconditionnement ischémique ou hypoxique. ${ }^{1,2}$ C'est un message naturel qui dit que «ce qui ne vous tue pas vous rend plus forts.» Il y a un certain nombre d'implications biologiques importantes à ce phénomène. D'abord, les avantages thérapeutiques potentiels du préconditionnement peuvent être sollicités quand on s'attend à des stress physiologiques. De plus, le préconditionnement est un outil intéressant pour étudier les mécanismes possibles de la mort cellulaire.

Le concept de la coagulation et de l'inflammation implique un équilibre finement réglé entre les profacteurs et les antifacteurs, les mécanismes qui conduisent à la mort cellulaire démontrent aussi cet équilibre. L'étude de la mort des cellules a généralement mis l'accent sur les profacteurs, probablement parce que la mort cellulaire est un paramètre relativement facile à mesurer. Toutefois, le préconditionnement est largement reconnu pour provoquer l'induction des facteurs cytoprotecteurs et il est, par conséquent, un outil important pour élucider les mécanismes antifacteurs ou cytoprotecteurs innés.

Les exemples de préconditionnement sont abondants. Ainsi, l'induction de robustes mécanismes cytoprotecteurs est impliquée dans de nombreux exploits spectaculaires de la nature comme la submersion prolongée des mammifères plongeurs et l'hibernation. En médecine clinique, les exemples de préconditionnement comprennent les effets protecteurs potentiels de l'angine préexistante sur la survie de patients qui subissent un infarctus du myocarde ${ }^{3}$ ou la valeur des courtes interruptions du flux coronaire pendant l'angioplastie coronaire percutanée qui précède le gonflement prolongé du ballonnet. ${ }^{4}$ Maintenant il existe toute une documentation établissant l'application chez l'humain du préconditionnement ischémique myocardique. ${ }^{5}$

Mis à part les anciens remèdes ou peut-être même l'homéopathie, la première démonstration de préconditionnement a été décrite en 1964 quand on a noté que l'hypoxie globale protégeait le métabolisme du cerveau pendant une hypoxie subséquente. ${ }^{6}$ Depuis lors, l'étude du préconditionnement s'est développée par des efforts pour créer et comprendre l'induction de la tolérance ischémique et hypoxique. Généralement, une expérience de préconditionnement comprend l'ischémie ou l'hypoxie de l'organe considéré, suivie de l'évaluation des effets d'une exposition subséquente à l'ischémie ou à l'hypoxie. L'interruption de certains mécanismes et la démonstration d'une diminution de la tolérance prouveraient alors que ce mécanisme est impliqué dans la cytoprotection endogène.

De nombreux mécanismes dommageables potentiels sont impliqués dans la lésion ischémique cérébrale. De ce fait, il y a de nombreuses sources de stress reliées, autres que l'ischémie/l'hypoxie, qui peuvent tour à tour induire la tolérance à l'ischémie/l'hypoxie. Par exemple, des articles mentionnent que l'hypothermie et l'hyperthermie, 7,8 l'inflammation, ${ }^{9}$ l'épilepsie, ${ }^{10}$ les radicaux libres d'oxygène $^{11,12}$ et l'oxygène hyperbare ${ }^{13,14}$ peuvent induire une tolérance ischémique ou hypoxique. Étant donné la nature entremêlée de nombreuses cascades concernées dans les lésions cérébrales ischémiques ou hypoxiques, l'induction de la tolérance à un stress relié à l'ischémie peut être amorcée au moyen d'un autre déclencheur. En outre, même un préconditionnement éloigné a été décrit, où la tolérance ischémique endothéliale coronaire a été augmentée à la suite d'une ischémie transitoire d'un membre, ${ }^{15}$ probablement par un effet qui modifiait l'activation polynucléaire neutrophile. ${ }^{16}$

Dans le présent numéro du Journal, Zhang et coll. ${ }^{17}$ présentent une expérience de préconditionnement accompagnée de quelques commentaires. De prime abord, il semble étrange d'induire un préconditionnement ischémique avec un apport d'oxygène enrichi plutôt que limité. Néanmoins, ce même groupe et d'autres ont déjà mentionné que l'hyperoxie et l'oxygène hyperbare sont certainement des déclencheurs du préconditionnement ischémique. En 
conséquence, une partie de l'objectif fixé et de la discussion de cet article est de déterminer la dose d'oxygène nécessaire pour déclencher le préconditionnement. Il existe un certain nombre d'extensions à leurs résultats. Que l'oxygène fonctionne comme un déclencheur de préconditionnement laisse à penser qu'il est nuisible ou toxique en quelque sorte. Cette affirmation cadre avec le problème bien décrit de la toxicité de l'oxygène du poumon, laquelle est reliée à la fois à la concentration et à la durée de l'exposition.

Les deuxièmes objectif expérimental et sujet de discussion portent sur la recherche du rôle des radicaux libres d'oxygène dans le processus de préconditionnement ischémique cérébral. Cela n'explique pas seulement le paradoxe apparent de l'hyperoxie qui devient un déclencheur de préconditionnement, mais cela souligne aussi l'importance des radicaux libres d'oxygène dans l'ischémie cérébrale et la cytoprotection innée possible par des antioxydants endogènes.

Dans l'article de Zhang et coll., on suggère que le préconditionnement soit déclenché par une dose d'oxygène de 1 atmosphère absolue (ATA) pendant 24 $\mathrm{h}$, un jour avant l'ischémie focale. Les auteurs introduisent l'idée que le dosage est crucial dans le déclenchement du préconditionnement ischémique. Ils rappellent que leur travail antérieur démontrait que c'est l'hyperoxie et non l'hyperbaricité de l'oxygène qui est critique. Ces éléments doivent toutefois être clarifiés. La pression partielle d'oxygène est le produit de la pression barométrique ambiante et de la fraction d'oxygène. Les auteurs mentionnent leur recherche antérieure où 2,5 ATA d'oxygène à $21 \%$ n'ont pas eu d'effet déclencheur de préconditionnement ischémique, tandis que 1 ATA d'oxygène à $100 \%$ en a eu un. Ils ont conclu que l'hyperbaricité n'était pas importante. Cependant, l'expérience comportait aussi une comparaison de différentes doses d'oxygène (pressions partielles de 0,5 vs 1,0 ATA). Dans le but de régler la question de la pression barométrique ambiante vs l'hyperoxie, on doit comparer des pressions partielles d'oxygènes égales, produites à différentes pressions ambiantes. Par exemple, 0,60 ATA d'oxygène en utilisant 1 ATA d'oxygène à $60 \%$ comparé à 3 ATA d'oxygène à $20 \%$. Une fois clarifiée toute la question de la baricité ambiante, le dosage peut être exprimé ensuite par la pression partielle d'oxygène (ATA obtenue par tous les moyens) et la durée.

En général, le dosage de cet effet déclencheur est intéressant pour diverses raisons. D'abord, comme le propose l'auteur, il pourrait avoir des implications thérapeutiques. Ensuite, il est important de décrire les caractéristiques d'un modèle de recherche pour pouvoir l'utiliser efficacement dans l'étude d'autres questions.
Finalement, la courbe dose-réponse elle-même peut nous renseigner sur le mécanisme et nous donner un point de vue sur la pertinence de ce phénomène (préconditionnement) suivant l'exposition à différentes doses (pressions d'oxygène). L'article de Zhang et coll. précise la dose d'oxygène à utiliser avec ce modèle.

L'application clinique du préconditionnement ischémique est certainement intéressante ; cependant, le dosage précis est critique. En utilisant un déclencheur comme l'hypoxie, le calibrage de la bonne dose pourrait être difficile à cause de l'écart étroit entre le seuil inférieur nécessaire et la limite supérieure d'une lésion cellulaire réelle. Il semble que l'usage d'une source d'oxygène enrichi pour déclencher le préconditionnement soit plus acceptable en clinique.

L'étude présente aussi la possibilité que, en plus d'être sensible à la toxicité pulmonaire, le cerveau soit sensible à des niveaux toxiques de radicaux libres d'oxygène, ce qui soulève la question de l'utilisation que nous faisons d'apport d'oxygène enrichi dans d'autres situations. Par exemple, pendant le sevrage de la circulation extracorporelle, au moment de l'occurrence plus élevée de micro-embolie et donc, de lésion ischémique cérébrale possible, les patients sont souvent traités avec des concentrations d'oxygène qui se rapprochent de 1,0 ATA. Est-ce le bon moment pour augmenter la production de radicaux libres d'oxygène dans le cerveau?

Zhang et coll. présentent des faits qui laissent croire que, dans leur étude, le déclencheur de préconditionnement implique la production de radicaux libres d'oxygène. Un piégeur de radicaux libres d'oxygène administré à des animaux pendant le préconditionnement prévient ce phénomène. L'expérience suggère donc que l'apport d'oxygène enrichi ait conduit à la production accrue de radicaux libres qui ont été le déclencheur du processus ultérieur de tolérance ischémique. Il y a cependant quelques étapes intermédiaires. D'autres études ${ }^{1}$ ont démontré que les déclencheurs sont détectés par des capteurs qui, à leur tour, emploient des mécanismes de transduction (comme l'expression de gène précoce immédiat, l'oxyde nitrique, la phosphorylation de la protéine tyrosine) dans le but d'influencer les effecteurs du préconditionnement [comme les protéines de choc thermique, les piégeurs de radicaux libres, la régulation positive des récepteurs de l'acide gamma-aminobutyrique (GABA) et la libération accrue de GABA, la libération du facteur de croissance vasculaire endothéliale ou du facteur de croissance BDNF (de l'anglais: brain derived neurotrophic factor)].

Même si l'usage de ce paradigme a permis de mieux comprendre la biopathologie de la mort cellulaire 
ischémique, de nombreuses questions doivent obtenir des réponses avant que nous puissions procéder à l'application clinique dans le cerveau. Ainsi, les mécanismes du préconditionnement sont divisés en phases immédiate et retardée. Le myocarde démontre assez bien un préconditionnement immédiat. Par ailleurs, le cerveau répond en phase retardée. Les paradigmes semblables au préconditionnement qui comportent des intervalles relativement courts entre la commande et l'agression conduisent en fait à des lésions cérébrales exacerbées. ${ }^{2}$ Et pour compliquer encore plus notre décision d'utiliser l'oxygène pendant la période péri-ischémique, des animaux exposés à 1,0 ATA d'oxygène pendant trois à six heures, suivant une ischémie globale, ont présenté un taux de mortalité beaucoup plus élevé. ${ }^{18}$ L'équilibre entre la chronologie des faits, les profacteurs et les antifacteurs ainsi que le dosage est donc critique et complexe, comme nous l'avons appris avec la manipulation de l'inflammation et de la coagulation. L'avancée de l'application clinique du préconditionnement ischémique cérébral suivra probablement l'élucidation des mécanismes déclencheur, capteur, transducteur et effecteur, et du potentiel de facilitation et de modulation des composantes individuelles.

\section{References}

1 Dirnagl U, Simon RP, Hallenbeck JM. Ischemic tolerance and endogenous neuroprotection. Trends Neurosci 2003; 26: 248-54.

2 Kirino T. Ischemic tolerance. J Cereb Blood Flow Metab 2002; 22: 1283-96.

3 Tomai F, Crea F, Chiariello L, Gioffre PA. Ischemic preconditioning in humans: models, mediators, and clinical relevance. Circulation 1999; 100: 559-63.

4 Yellon DM, Dana $A$. The preconditioning phenomenon. A tool for the scientist or a clinical reality? Circ Res 2000; 87: 543-50.

5 Kloner RA, Jennings RB. Consequences of brief ischemia: stunning, preconditioning, and their clinical implications. Part 1. Circulation 2001; 104: 2981-9.

6 Dabl NA, Balfour WM. Prolonged anoxic survival due to anoxia pre-exposure: brain ATP, lactate, and pyruvate. Am J Physiol 1964; 207: 452-6.

7 Chopp M, Chen H, Ho KL, et al. Transient hyperthermia protects against subsequent forebrain ischemic cell damage in the rat. Neurology 1989; 39: 1396-8.

8 Nishio S, Yunoki M, Chen ZF, Anzivino MJ, Lee KS. Ischemic tolerance in the rat neocortex following hypothermic preconditioning. J Neurosurg 2000; 93 : 845-51.

9 Zimmermann C, Ginis I, Furuya K, et al. Lipopolysaccharide-induced ischemic tolerance is asso- ciated with increased levels of ceramide in brain and in plasma. Brain Res 2001; 895: 59-65.

10 Blondeau N, Widmann C, Lazdunski M, Heurteaux C. Polyunsaturated fatty acids induce ischemic and epileptic tolerance. Neuroscience 2002; 109: 231-41.

11 Buttke TM, Sandstrom PA. Oxidative stress as a mediator of apoptosis. Immunol Today 1994; 15: 7-10.

12 Ravati A, Ablemeyer B, Becker A, Klumpp S, Krieglstein $J$. Preconditioning-induced neuroprotection is mediated by reactive oxygen species and activation of the transcription factor nuclear factor- $\kappa \mathrm{B}$. J Neurochem 2001; 78: 909-19.

13 Dong H, Xiong L, Zhu Z, Chen S, Hou L, Sakabe T. Preconditioning with hyperbaric oxygen and hyperoxia induces tolerance against spinal cord ischemia in rabbits. Anesthesiology 2002; 96: 907-12.

14 Wada K, Miyazawa T, Nomura N, Tsuzuki N, Nawashiro H, Shima K. Preferential conditions for and possible mechanisms of induction of ischemic tolerance by repeated hyperbaric oxygenation in gerbil hippocampus. Neurosurgery 2001; 49: 160-7.

15 Kharbanda RK, Mortensen UM, White PA, et al. Transient limb ischemia induces remote ischemic preconditioning in vivo. Circulation 2002; 106: 2881-3.

16 Kharbanda RK, Peters $M$, Walton $B$, et al. Ischemic preconditioning prevents endothelial injury and systemic neutrophil activation during ischemia-reperfusion in humans in vivo. Circulation 2001; 103: 1624-30.

17 Zhang $X$, Xiong L, Hu W, et al. Preconditioning with prolonged oxygen exposure induces ischemic tolerance in the brain via oxygen free radical formation. Can J Anesth 2004; 51: 258-63.

18 Mickel HS, Vaishnav YN, Kempski O, von Lubitz D, Weiss JF, Feuerstein G. Breathing 100\% oxygen after global brain ischemia in mongolian gerbils results in increased lipid peroxidation and increased mortality. Stroke 1987; 18: 426-30. 